\title{
Finite element network approximation of conductivity in particle composites
}

\author{
Daniel Peterseim • Carsten Carstensen
}

\begin{abstract}
A new finite element method computes conductivity in some unstructured particle-reinforced composite material. The 2-phase material under consideration is composed of a poorly conducting matrix material filled by highly conducting circular inclusions which are randomly dispersed. The mathematical model is a Poisson-type problem with discontinuous coefficients. The discontinuities are huge in contrast and quantity. The proposed method generalizes classical continuous piecewise affine finite elements to special computational meshes which encode the particles in a network structure. Important geometric parameters such as the volume fraction are preserved exactly. The computational complexity of the method is (almost) proportional to the number of inclusions. This is minimal in the sense that the representation of the underlying geometry via the positions and radii of the inclusions is of the same complexity. The discretization error is proportional to the distance of neighboring inclusions and independent of the conductivity contrast in the medium.
\end{abstract}

Mathematics Subject Classification (2000) $\quad 65$ N15 $\cdot$ 65N30 $\cdot 74$ Q20

The work of D. Peterseim was supported by the DFG Research Center Matheon Berlin through project C33.

D. Peterseim $(\varangle) \cdot$ C. Carstensen

Institut für Mathematik, Humboldt-Universität zu Berlin,

Unter den Linden 6, 10099 Berlin, Germany

e-mail: peterseim@math.hu-berlin.de

C. Carstensen

Department of CSE, Yonsei University, Seoul, Korea 


\section{Introduction}

Composite materials (or composites for short) are engineered materials made from two or more constituents with significantly different physical properties. In a typical configuration, randomly distributed filler particles (inclusions) are surrounded by a second material (matrix) which binds the filler particles together.

The numerical simulation of material properties aims at a better understanding how conductivity depends on controllable variables (e.g., thermal conductivities of the material components, relative volumes, and particles shapes) and hence provides the opportunity to develop materials with enhanced performance for the particular application.

The design of efficient and reliable numerical methods for such problems is challenging. The complexity of the underlying geometry makes classical approaches hardly feasible (cf. Sect. 1.2); in the typical geometric setting, the inclusions are too big for any perturbation analysis or homogenization method, and they are too many or they are packed too densely to resolve them easily with standard finite element meshes. We face this difficulty even for simple continuum models of some material property of interest, e.g. the linear elliptic model problem of heat conduction considered in this paper (see Sect. 1.1).

Based on an efficient treatment of the microscopic geometry, the new method described in this paper (cf. Sect. 1.3) allows reliable numerical simulation of the model problem with many inclusions independent of the degree of disorder in the geometry.

\subsection{Model problem}

This paper considers a representative 2-dimensional model of a particle-reinforced composite occupying the nonempty open bounded convex polyhedral domain $\Omega \subset$ $\mathbb{R}^{2}$. Let $\mathcal{B}_{\text {inc }}$ be a set of closed, pairwise disjoint disks of positive radii (inclusions) contained in a domain $\Omega \subset \mathbb{R}^{2}$, i.e.,

$$
B \subset \Omega \text { and } \operatorname{dist}(B, \tilde{B})>0 \text { for all } B, \tilde{B} \in \mathcal{B}_{\text {inc }} \text { with } B \neq \tilde{B} \text {. }
$$

In the present context, the number $N:=\# \mathcal{B}_{\text {inc }}$ of inclusions is a very large parameter. The two material phases are represented by the union of the inclusions $\Omega_{\mathrm{inc}}$, and by the so called matrix (the perforated domain) $\Omega_{\text {mat }}$,

$$
\Omega_{\mathrm{inc}}:=\bigcup_{B \in \mathcal{B}_{\mathrm{inc}}} \operatorname{int}(B) \text { and } \Omega_{\mathrm{mat}}:=\Omega \backslash \bar{\Omega}_{\mathrm{inc}} .
$$

The outer boundary $\Gamma:=\partial \Omega$ is partitioned into two parts $\Gamma_{D}$ and $\Gamma_{N}$, where $\Gamma_{D}$ is closed and has a positive surface measure while its relative complement $\Gamma_{N}:=\Gamma \backslash \Gamma_{D}$ is relatively open, and the number of contact points $\Gamma_{D} \cap \bar{\Gamma}_{N}$ is finite.

The material geometry enters the problem through a coefficient function $c \in$ $L^{\infty}(\Omega)$ which jumps between the material components. For simplicity $c$ is chosen to 
be constant with respect to each of the two phases and normalized with respect to the matrix material, i.e.,

$$
c(x)= \begin{cases}1 & \text { if } x \in \Omega_{\text {mat }} \\ c_{\text {cont }} & \text { if } x \in \Omega_{\text {inc }}\end{cases}
$$

The constant $c_{\text {cont }} \geq 1$ represents the conductivity contrast in the medium.

Consider the set of admissible temperature distributions

$$
\mathcal{A}:=u_{D}+V \text { with } V:=\left\{u \in H^{1}(\Omega) \mid u=0 \text { on } \Gamma_{D}\right\}
$$

for $u_{D} \in H^{1}(\Omega) \cap C^{0}(\bar{\Omega})$. Given some force density $f \in V^{*}$, the effective conductivity of the composite

$$
c_{\text {eff }}:=\min _{u \in \mathcal{A}} \mathfrak{E}(u)
$$

minimizes the energy functional $\mathfrak{E}$,

$$
\mathfrak{E}(v):=\frac{1}{2} \int_{\Omega} c(x)|\nabla v(x)|^{2} \mathrm{~d} x-\int_{\Omega} f(x) v(x) \mathrm{d} x \text { for all } v \in H^{1}(\Omega) .
$$

\subsection{Challenges to numerical simulations}

In practical applications, the parameter $c_{\text {cont }} \gg 1$ is very large. In addition, the coefficient function, which is the output of certain (random) production processes (e.g. mixing of the particles within a liquid matrix material followed by hardening), has to be regarded as a statistical parameter. Corresponding to Berlyand [3], the latter two issues, random micro-structures on multiple scales and high contrast in physical properties, are the two characteristic features of general composites. They lead to major difficulties for a numerical approximation of problem (1.2).

Classical FEM A classical method for the approximate solution of (1.2) is the finite element method. However, in the present context, standard finite element approaches suffer from the fact that the material interface $\partial \Omega_{\text {inc }}$ needs to be resolved by the underlying mesh in order to get satisfactory results. The required resolution of the coefficient geometry forces even the coarsest available meshes to be very fine, i.e., the minimal mesh size has to be at most of order of the inclusion radii. Additionally, finite element methods often require high quality meshes (shape regularity) which puts even more constraints on mesh generation. Thus, the minimal number of nodes in a reasonable mesh depends critically on the distribution of the holes and their distances; Fig. 1 illustrates the problem in a model situation, which is eased for visualization purposes.

Minimal complexity Since the underlying geometry is of stochastic nature problem (1.2), typically, needs to be solved many times for different coefficient configurations 

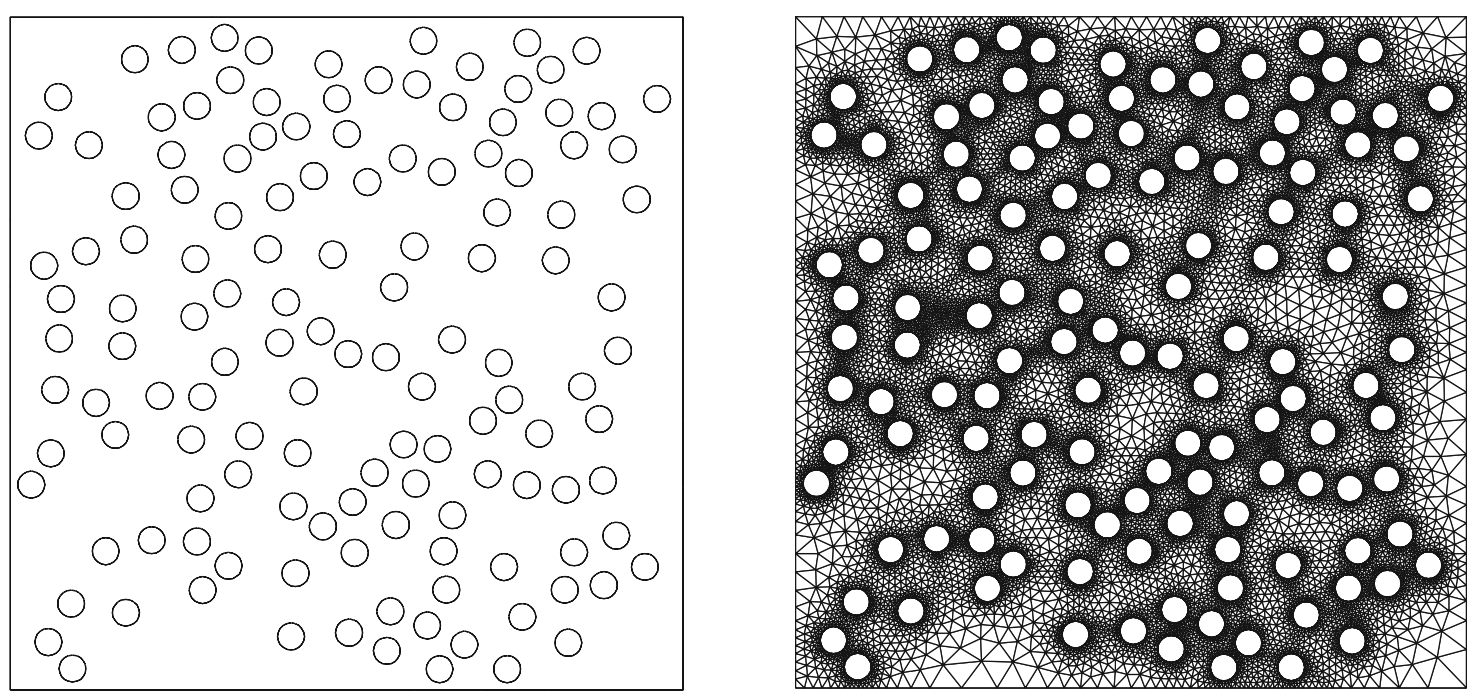

Fig. 1 Model domain (unit square) containing 133 circular inclusions with radius $r=0.02$ (left) and "coarse" shape regular triangulation with 33903 elements (right)

within a statistical investigation of material properties (by a Monte Carlo method). For example, the accuracy of the approximation of the expected temperature distribution subject to a to random distribution of particles in the material, is of order $M^{-1 / 2}$, where $M$ denotes the number of samples. Since the coefficient is different for different samples, meshes cannot be re-used but need to be re-computed for every single sample of the particle distribution. Hence, the computation of the finite element mesh is crucial in all complexity discussions and cannot be neglected as a precomputation (cf. Fig. 1). With regard to the possibly huge number of instances of problem (1.2) that need to be considered, this paper aims at a reasonable discrete model of minimal complexity. Minimality is determined by the data of the problem and therefore mainly by its geometry. The geometry representation requires storing the pairs of centers and radii of the $N$ inclusions (the complexity of the representation of the outer boundary is supposed to be small compared to $N$ ). A model is considered to have minimal complexity if it provides an approximate solution in time and space complexity $\mathcal{O}(N)$. The finite element method to be presented in this paper satisfies the complexity requirement up to logarithmic factors (cf. Section below).

\subsection{The new structural finite element approach}

In this paper ideas from network approximations [3-5,19] are combined with nonstandard finite element methods to derive a new structural finite element method of almost minimal complexity. In particular, a special geometry treatment inspired by networks is combined with the flexibility of finite element methods. As in discrete network methods, the inclusions are modeled in a network structure. They appear as elements of the computational mesh, supplemented by channel-like objects that connect neighboring inclusions and, finally, triangles. The mesh generalizes standard Delaunay triangulations of points in the plane to sets of disks. It can be computed and represented efficiently. A generalization of continuous first-order finite ele- 
ments based on the new, problem-adapted subdivisions is introduced. Its realization is conceivably simple and it provides accurate numerical approximations at almost minimal complexity. More precisely, for the solution $u \in \mathcal{A} \cap H^{2}\left(\Omega_{\text {mat }} \cup \Omega_{\text {inc }}\right)$ of (1.2) and its structural finite element approximation $u_{S}$ it holds (see Theorem 3.1, Corollaries 3.1 and 3.2).

$$
\left\|\sqrt{c} \nabla\left(u-u_{S}\right)\right\|_{L^{2}(\Omega)} \leq C_{f, u_{D}, \mathcal{B}_{\text {inc }}}\|h\|_{L^{\infty}(\Omega)},
$$

where $h$ is a local mesh size parameter. The constant does not depend on contrast. Its dependencies on the geometry of the material (e.g., touching inclusions) are discussed in detail.

The overall motivation for the novel network approximation is its optimal complexity in the sense that the cost for a meaningful approximation remains proportional to the number of inclusions.

\subsection{Numerical upscaling}

The number of degrees of freedom might be reduced further by using multiscale methods, e.g., [7,11,16,17,20,21]. These methods are based on arbitrary coarse meshes that, more or less, ignore the geometric scales of the coefficient. The influence of the coefficient is instead coded in the finite element basis functions or some modified discrete operator. For this, multiscale methods require some preprocessing that involves the solution of the original problem on subdomains. The solution of these local problems, however, faces the same difficulties as the original problem, i.e., it requires submeshes fine enough to capture the heterogeneities (the influence of the microscopic geometry on macroscopic material properties can only be studied if the microscopic geometry enters the discretization). In this regard, the method presented here might be employed as an efficient fine scale solver within some multiscale numerical framework.

\subsection{Outline}

Section 2 defines a problem adapted generalization of triangular meshes modeling the inclusions as (vertex-like) elements of a subdivision. Based on this new type of meshes a generalized nodal basis defining a generalized conforming first-order approximation space is introduced. Contrast-independent a priori error estimates for the proposed new finite element method are given in Sect. 3. Section 4 discusses open problems and future generalizations of the method.

\subsection{Notation}

In this paper, capital letters $A, B, C, \ldots$ indicate sets. Calligraphic capital letters $\mathcal{B}, \mathcal{P}, \ldots$ denote sets of sets. For a given set of sets $\mathcal{B}$ the union of its elements is denoted by $\cup \mathcal{B}:=\bigcup_{B \in \mathcal{B}} B$. Basic topological notations are used: For any subset $X$ 
of a metric space its closure is denoted by $\bar{X}$, its interior by $\operatorname{int}(X)$, and its boundary by bnd $(X)$. In what follows, $\operatorname{dist}(\cdot, \cdot)$ denotes the Euclidean distance in $\mathbb{R}^{2}$. The measure $|\cdot|$ is context-sensitive and refers to the volume of a set relative to its dimension, i.e., $|\cdot|$ denotes the length of a curve, or the area of a domain. The distance between nonempty subsets $A, B \subset \mathbb{R}^{2}$ reads

$$
\operatorname{dist}(A, B):=\inf _{x \in A, y \in B} \operatorname{dist}(x, y)
$$

Given some bounded domain $\Omega$, standard notation for (fractional) Sobolev spaces $W_{p}^{m}(\Omega), m \geq 0, p \in \mathbb{N} \cup\{0\}$, and their corresponding norms $\|\cdot\|_{W_{p}^{m}(\Omega)}$ and seminorms $|\cdot|_{W_{p}^{m}(\Omega)}$ is used; $H^{m}(\Omega)$ abbreviates $W_{2}^{m}(\Omega)(m \in \mathbb{N})$ and $L^{p}(\Omega)$ abbreviates $W_{p}^{0}(\Omega)$. Given two disjoint bounded Lipschitz domains $\Omega_{1}$ and $\Omega_{2}$, the space $H^{m}\left(\Omega_{1} \cup \Omega_{2}\right)$ denotes the space of all functions $u \in L^{2}\left(\Omega_{1} \cup \Omega_{2}\right)$ with $\left.u\right|_{\Omega_{1}} \in H^{m}\left(\Omega_{1}\right)$ and $\left.u\right|_{\Omega_{2}} \in H^{m}\left(\Omega_{2}\right)$. The dual space of a Hilbert space $V$ is indicated by $V^{*}$. The space of $\mathbb{R}$-valued continuous functions on a set $\Omega$ is denoted by $C^{0}(\Omega)$.

\section{A minimal conforming finite element space}

This section introduces a conforming finite element space which can be regarded as a generalization of the classical continuous piecewise affine finite element space on a special mesh.

\subsection{Geometric preliminaries}

Cyclic polygons A convex polygon $T$ is the closed convex hull of 2 or more distinct points. The set of vertices (corners) $\mathcal{V}(T)$ is the minimal set of points $x_{1}, x_{2}, \ldots, x_{k} \in$ $\mathbb{R}^{2}$, such that $T=\operatorname{conv}\left(\left\{x_{1}, x_{2}, \ldots, x_{k}\right\}\right)$. According to the number of its vertices, a convex polygon is denoted as a convex $k$-gon. The boundary of a convex $k$-gon can be described by the union of at most $k$ line segments called edges. The set of edges of a convex polygon $T$ is denoted by $\mathcal{E}(T)$. A convex polygon $T$ is called cyclic if its vertices (corners) $V(T)$ are located on the boundary of a (closed) disk $C D=C D(T)$ which is denoted as the circumdisk of $T$. Examples of cyclic polygons are line segment, triangles, or rectangles.

Infinite Delaunay Triangulations A regular (possibly infinite) triangulation of a domain $\Omega \subset \mathbb{R}^{2}$ into cyclic polygons is a set of cyclic polygons $T$ such that

$$
\cup \mathcal{T}=\bar{\Omega}
$$

and any two distinct cyclic polygons are either

a) disjoint, $T_{1} \cap T_{2}=\emptyset$, or

b) share exactly one vertex $z, T_{1} \cap T_{2}=V\left(T_{1}\right) \cap V\left(T_{2}\right)=\{z\}$, or

c) have one edge $E=\operatorname{bnd}\left(T_{1}\right) \cap \operatorname{bnd}\left(T_{2}\right)=\mathcal{E}\left(T_{1}\right) \cap \mathcal{E}\left(T_{2}\right)$ in common. 
The set of all edges resp. vertices of a triangulation $\mathcal{T}$ is written as

$$
\mathcal{E}(\mathcal{T}):=\bigcup_{T \in \mathcal{T}} \mathcal{E}(T) \text { resp. } \mathcal{V}(\mathcal{T}):=\bigcup_{T \in \mathcal{T}} \mathcal{V}(T)
$$

A regular triangulation $\mathcal{T}$ is called Delaunay [10] if every element $T \in \mathcal{T}$ satisfies the Delaunay criterion

$$
C D(T) \cap \mathcal{V}(\mathcal{T})=\mathcal{V}(T),
$$

that is, the circumdisc of $T$ does not contain any vertices of $\mathcal{T}$ except those of $T$. Given a set of vertices $\mathcal{V}$, the Delaunay triangulation of $\operatorname{conv}(\mathcal{V})$ is uniquely determined (if cyclic polygons are considered). It can be constructed, e.g., by exploiting duality with respect to the Voronoi diagram [27] of $\mathcal{V}$. The uniqueness is due to the consideration of cyclic polygons instead of just triangles. In the subsequent paragraph, cyclic $k$-gons with $k>3$ will further be decomposed into triangles.

\subsection{Geometric modeling of particle composites}

The geometry of model problem (1.2) is represented by a finite set $\mathcal{B}$ of closed disks. Every $B \in \mathcal{B}$ is described by its center $c_{B}=\operatorname{mid}(B)$ and its radius $r_{B}=\operatorname{diam}(B) / 2 \geq$ 0 . The elements of $\mathcal{B}$ are denoted as generalized vertices and partitioned into the two subsets $\mathcal{B}_{\text {inc }}$ and $\mathcal{B}_{\text {mat }}$, i.e.,

$$
\mathcal{B}=\mathcal{B}_{\text {inc }} \cup \mathcal{B}_{\text {mat }} \text { and } \mathcal{B}_{\text {inc }} \cap \mathcal{B}_{\text {mat }}=\emptyset .
$$

The set $\mathcal{B}_{\text {inc }}$ contains the inclusions of model problem (1.2), i.e., closed disks of positive radius. The set $\mathcal{B}_{\text {mat }}$ contains closed disks of radius zero with

$$
\operatorname{conv}\left(\cup \mathcal{B}_{\text {mat }}\right)=\bar{\Omega} \text { and } \Gamma_{D} \cap \bar{\Gamma}_{N} \subset \cup \mathcal{B}_{\text {mat }} .
$$

Thus $\mathcal{B}_{\text {mat }}$ contains the corners of $\partial \Omega$ and all points where the type of boundary condition switches between Dirichlet and Neumann; but $\mathcal{B}_{\text {mat }}$ might contain additional points (disks with vanishing radii) in the interior of the matrix $\Omega_{\text {inc }}$, which offers the possibility of refinement and increased local resolution within the finite element framework.

By $\mathcal{T}_{\text {mat }}$ we denote the Delaunay triangulation of $\Omega_{\text {mat }}$ such that

$$
\mathcal{V}\left(\mathcal{T}_{\text {mat }}\right)=\mathcal{B}_{\text {mat }} \cup \bigcup_{B \in \mathcal{B}_{\text {inc }}} \partial B
$$

Figure 2 displays a detail of $\mathcal{T}_{\text {mat }}$ for some set of disks $\mathcal{B}$. Obviously, $\mathcal{T}_{\text {mat }}$ consists of two classes of cyclic polygons (see [24]), namely,

a) (possibly infinitely many) cyclic 2-gons $\mathcal{T}_{\text {mat }}^{1}$, i.e., line segments whose vertices are located on the circumference of exactly two distinct disks, and 


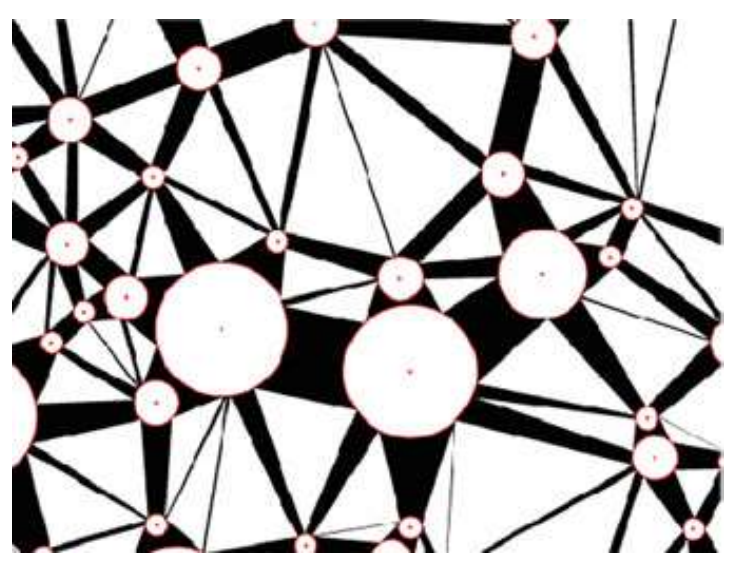

(a) Generalized Delaunay triangulation

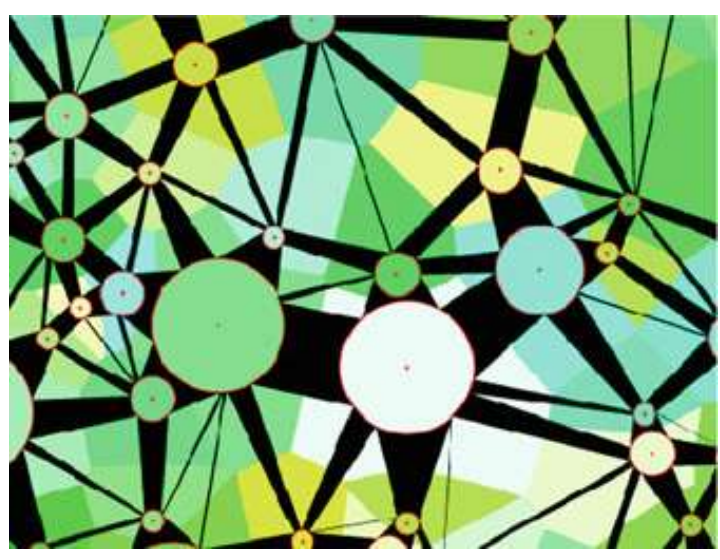

(b) Genrealized Delaunay triangulation and dual Voronoi tessellation

Fig. 2 Generalized Delaunay triangulation with respect to disks in the plane

b) (finitely many) cyclic $k$-gons $\mathcal{T}_{\text {mat }}^{\Delta}$ for $k \geq 3$.

For simplicity we assume that $\mathcal{T}_{\text {mat }}^{\Delta}$ contains exclusively triangles. This assumption can always be fulfilled if we consider a triangulation $\tilde{\mathcal{T}}_{\text {mat }}$ in which the $4,5, \ldots$-gons of $\mathcal{T}_{\text {mat }}$ are further decomposed into triangles; $\tilde{\mathcal{T}}_{\text {mat }}$ is not Delaunay in the sense of (2.1) but fulfills the weaker Delaunay criterion

$$
\operatorname{int}(C D(T)) \cap \mathcal{V}\left(\tilde{\mathcal{T}}_{\text {mat }}\right)=\varnothing \quad \text { for all } T \in \tilde{\mathcal{T}}_{\text {mat }},
$$

that is, there are no vertices of $\tilde{\mathcal{T}}_{\text {mat }}$ in the interior of the circumdisk of $T \in \tilde{\mathcal{T}}_{\text {mat }}$. The subset $\mathcal{T}_{\text {mat }}^{\Delta}$ of triangles of $\mathcal{T}_{\text {mat }}$ provides structural (combinatorial) information about the set of inclusions $\mathcal{B}_{\text {inc }}$. It induces a neighborhood relation $\mathcal{N} \subset \mathcal{B}_{\text {inc }} \times \mathcal{B}_{\text {inc }}$ defined by the rule: $\left(B_{1}, B_{2}\right) \in \mathcal{N}$ if there exists a $T \in \mathcal{T}_{\text {mat }}^{\Delta}$ such that $V(T) \subset B_{1} \cup B_{2}$ and $V(T) \cap B_{1} \neq \emptyset$ and $V(T) \cap B_{2} \neq \emptyset$. For every pair $\left(B_{1}, B_{2}\right) \in \mathcal{N}$ of neighboring disks we define the channel-like object (a bundle of line segments)

$$
E\left(B_{1}, B_{2}\right):=\cup\left\{T \in \mathcal{T}_{\text {mat }}: V(T) \subset B_{1} \cup B_{2}\right\}
$$

Since $E\left(B_{1}, B_{2}\right)$ is an object that connects exactly two generalized vertices (disks) we denote $E\left(B_{1}, B_{2}\right)$ a generalized edge.

A finite subdivision $\mathcal{G}$ of $\Omega$, which will serve as the finite element mesh later, is given by

$$
\mathcal{G}=\mathcal{B}_{\text {inc }} \cup \mathcal{E} \cup \mathcal{T}
$$

where $\mathcal{B}_{\text {inc }}$ is the given set of disks, $\mathcal{E}:=\left\{E\left(B_{1}, B_{2}\right):\left(B_{1}, B_{2}\right) \in \mathcal{N}\right\}$ is the set of generalized edges and $\mathcal{T}:=\mathcal{T}_{\text {mat }}^{\Delta}$ is the set of triangles.

Remark 1 a) The subdivision $\mathcal{G}$ can be regarded as a generalization of classical Delaunay triangulations in the sense that disks might assume the classical role of vertices while edges (i.e., objects that connect two neighboring vertices) might 
generalize to channels. In the special case of equally sized inclusions such subdivisions have been used in discrete network approximations [3]. Apart from minor technical details regarding the treatment of element boundaries, the subdivision $\mathcal{G}$ fits into the framework of generalized Delaunay partitions for multidimensional sets of convex inclusions introduced in [23].

b) The subdivision $\mathcal{G}$ covers $\Omega$ while the intersection of any two of its elements is of measure zero.

c) The number of elements in $\mathcal{G}$ is proportional to the cardinality of $\mathcal{B}$ and thus is quasi minimal.

d) There is a duality concept which links generalized Delaunay triangulations to Voronoi tessellations with respect to the set of disks (see Fig. 2(b) and the next subsection). It generalizes straight-line duality between classical Voronoi tessellation and Delaunay triangulation of point sets. We refer to [23] for more insights about geometric duality and further references.

e) The generalized Delaunay triangulation $\mathcal{D}$ can be computed fast as explained subsequently. There exist algorithms of order $\mathcal{O}(\# \mathcal{B} \times \log (\# \mathcal{B})$ ) for the computation of Voronoi diagrams with respect to a set of disks $\mathcal{B}$; see, e.g., $[13,14,18]$. These algorithms, by duality, can also be employed for the computation of the generalized Delaunay subdivision.

We refer to the recent preprint [12] for an algorithmic presentation of this construction.

\subsection{Element parametrization and local mesh size}

The generalized vertices $\mathcal{B}_{\text {inc }}$ and the triangles $\mathcal{T}$ form affine families and can easily be represented by reference elements and affine mappings.

A parametrization of a generalized edge can be given as follows. Let $E=E\left(B_{1}, B_{2}\right)$ in $\mathcal{E}$ be a generalized edge that connects two generalized vertices $B_{1}, B_{2} \in \mathcal{B}$ and let

$$
\Sigma_{E}:=\left\{y \in \mathbb{R}^{2}: \operatorname{dist}\left(y, B_{1}\right)=\operatorname{dist}\left(y, B_{2}\right) \text { and } \operatorname{dist}\left(y, B_{1}\right) \leq \operatorname{dist}\left(y, \mathcal{B} \backslash\left\{B_{1}, B_{2}\right\}\right)\right\}
$$

denote the corresponding dual Voronoi edge, the set of points with equal distance to both $B_{1}$ and $B_{2}$. Without loss of generality we assume $r_{B_{1}} \geq r_{B_{2}}, c_{B_{1}}=(0,0), c_{B_{2}}=$ $(0, \delta), \delta>0$. Note that the Voronoi dual edge might not be connected (see Fig. 4a). The same applies to the generalized edge as it can be seen in Fig. 4b. We denote the number of connected components of $E$ by $K(E)$. The projection $\pi_{B_{1}}:=\operatorname{argmin}_{y \in B_{1}} \operatorname{dist}(\cdot, y)$ defines angles

$$
-\frac{\pi}{2} \leq \alpha_{E}^{1} \leq \beta_{E}^{1}<\alpha_{E}^{2} \leq \beta_{E}^{2}<\cdots<\alpha_{E}^{K(E)} \leq \beta_{i j}^{K(E)} \leq \frac{\pi}{2}
$$

such that

$$
\pi_{B_{1}}\left(\Sigma_{E}\right)=\bigcup_{k=1}^{K(E)} r_{B_{1}}\left[\sin \left(\left[\alpha_{E}^{k}, \beta_{E}^{k}\right]\right), \cos \left(\left[\alpha_{E}^{k}, \beta_{E}^{k}\right]\right)\right]^{T} .
$$


In other words, the parameters $\alpha_{E}^{1}, \ldots, \alpha_{E}^{K(E)}, \beta_{E}^{1}, \ldots, \beta_{E}^{K(E)}$ are the angular values of the projections of the Voronoi vertices which are connected by $\Sigma_{E}$, onto $B_{1}$. Those Voronoi vertices are simply the circumcenters of triangles adjacent to $E$. With the reference element

$$
\left.E^{\mathrm{ref}}=E^{\mathrm{ref}}\left(B_{1}, B_{2}\right):=(] \alpha_{E}^{1}, \beta_{E}^{1}[\cup \cdots \cup] \alpha_{E}^{K(E)}, \beta_{E}^{K(E)}[) \times\right] 0,1[,
$$

the mapping $J_{E}: E^{\mathrm{ref}} \rightarrow \operatorname{int} E$, given by

$$
\begin{aligned}
J_{E}(s, \lambda) & =(1-\lambda) r_{B_{1}}\left(\begin{array}{c}
\sin (s) \\
\cos (s)
\end{array}\right)+\lambda \pi_{B_{2}}\left(\left(\pi_{B_{1}} \mid \Sigma_{E}\right)^{-1}\left(r_{B_{1}}\left(\begin{array}{c}
\sin (s) \\
\cos (s)
\end{array}\right)\right)\right) \\
& =\left(\begin{array}{l}
\left((1-\lambda) r_{B_{1}}+\lambda r_{B_{2}}\right) \sin (s) \\
\left((1-\lambda) r_{B_{1}}-\lambda r_{B_{2}}\right) \cos (s)+\delta \lambda
\end{array}\right),
\end{aligned}
$$

parametrizes $E$. Figure 3 a visualizes the mapping $J_{E}$. Note that a generalized edge $E\left(B_{1}, B_{2}\right)$ is uniquely determined by the inclusion centers and radii, and the values of $\alpha_{E}, \beta_{E}$, and $\delta$.

The projection $\pi_{B_{1}, B_{2}}(\cdot):=\pi_{B_{2}}\left(\pi_{B_{1}}^{-1}(\cdot)\right)$ may be rewritten as

$$
\pi_{B_{1}, B_{2}}(x):=\underset{y \in \partial B_{2}}{\operatorname{argmin}} \frac{\operatorname{dist}(x, y)}{\max \left\{\left\langle(y-x) /\|y-x\|, v_{B_{1}}(y)\right\rangle, 0\right\}},
$$

where $v_{B_{1}}$ denotes the outer normal of $B_{1}$.

With

$$
H(s):=\frac{\left(\delta^{2}-2 \cos (s) \delta r_{B_{1}}\right)+r_{B_{1}}^{2}-r_{B_{2}}^{2}}{\left(2 r_{B_{2}}-2 r_{B_{1}}\right)+2 \delta \cos (s)}
$$

the parametrization $J_{E}$ assumes the form

$$
\begin{aligned}
J_{E}(s, \lambda)= & \left((1-\lambda) r_{1}+\lambda r_{2} \frac{r_{B_{1}}+H(s)}{r_{B_{2}}+H(s)}\right)\left[\begin{array}{l}
\sin (s) \\
\cos (s)
\end{array}\right] \\
& +\delta \lambda\left(1-\frac{r_{B_{2}}}{r_{B_{2}}+H(s)}\right)\left(\begin{array}{l}
0 \\
\delta
\end{array}\right) .
\end{aligned}
$$

We finally introduce some $\left(\mathcal{T}_{\text {mat }} \cup \mathcal{B}\right)$-piecewise constant meshsize function $h$ : $\Omega \rightarrow] 0, \infty[$ by

$$
\left.h\right|_{K}=h_{K}:=\operatorname{diam}(K) \text { for } K \in \mathcal{T}_{\text {mat }} \cup \mathcal{B}
$$

to be used in the forthcoming finite element analysis. Note that $h$ is not constant with respect to a generalized edge (of positive measure) but captures the distance between neighboring inclusions (see (2.4)). 


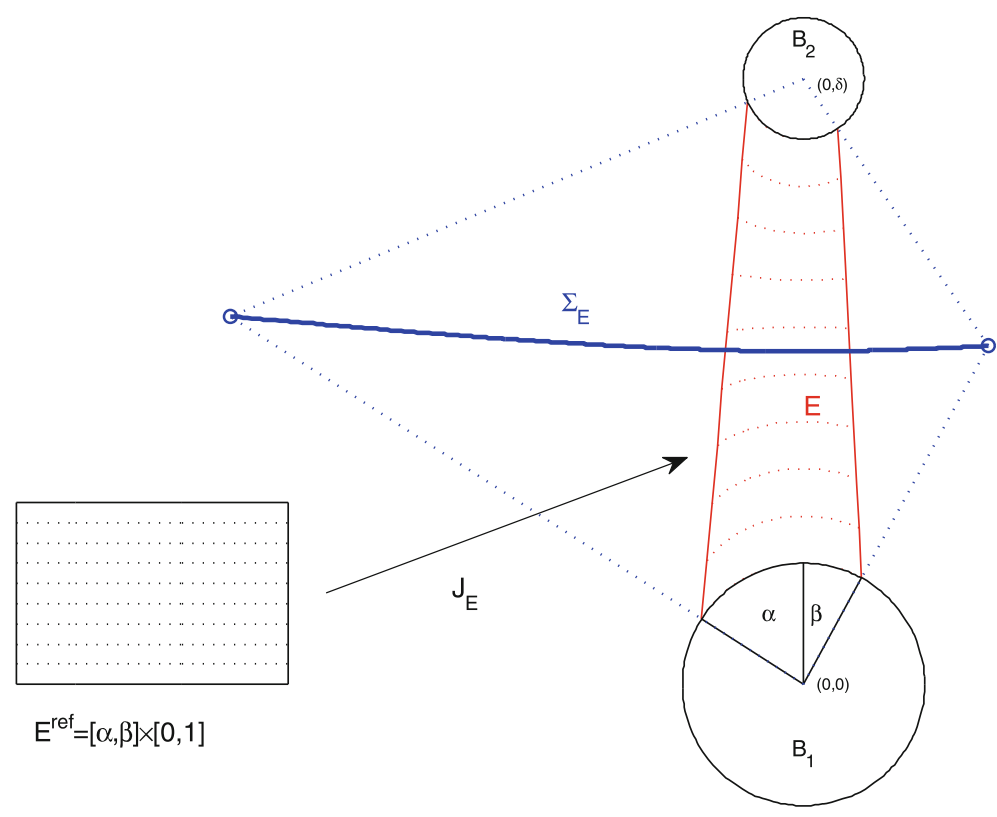

(a) Parametrization (isolines) of a generalized edge $E$ that connects two generalized vertices $B_{1}=\left\{x \in \mathbb{R}^{2} \mid\|x\| \leq r_{B_{1}} \|\right.$ and $B_{2}=\{x \in$ $\mathbb{R}^{2} \mid\|x-(0, \delta)\| \leq r_{B_{2}} \| ; \delta=1, r_{B_{1}}=0.2, r_{B_{2}}=0.1, \alpha_{E}=-1$, $\beta_{E}=0.5, K(E)=1$

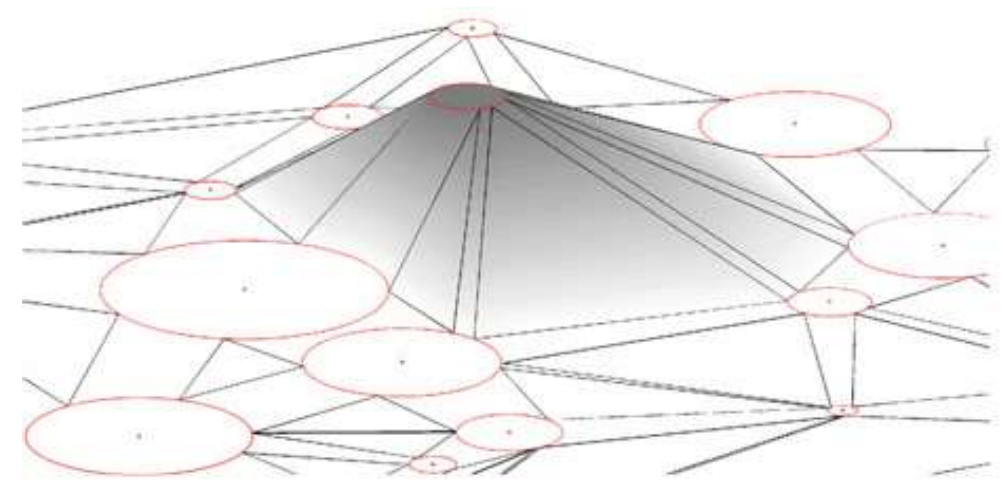

(b) A generalized nodal basis function taking value 1 on one node and zero on all the others

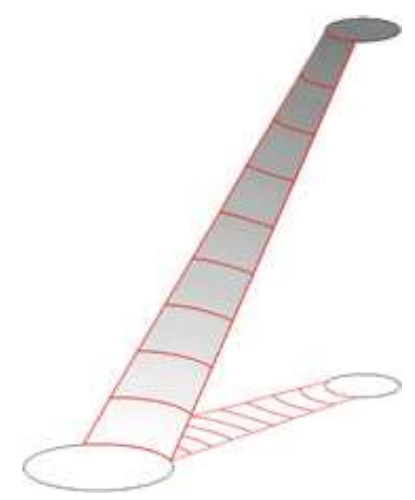

(c) Nodal basis function restricted to a generalized edge

Fig. 3 Edge parametrization and nodal basis function

\subsection{Finite element spaces}

The degrees of freedom of the finite element spaces are assigned to the entries of $\mathcal{B}$. Every $B \in \mathcal{B}$ defines a (local) $\mathcal{T}_{\text {mat }}$-affine basis function $\lambda_{B}: \mathbb{R}^{2} \rightarrow[0,1]$ with

$$
\lambda_{B} \equiv 1 \quad \text { in } B \quad \text { while } \lambda_{B} \equiv 0 \quad \text { in } \Omega_{\text {inc }} \backslash B .
$$

More precisely, $\lambda_{B}$ is unique continuous function with constant values on the inclusions as above and whose restriction to each element $T \in \mathcal{T}_{\text {mat }}$ is affine. This means that $\lambda_{B}$ is affine on all triangles $T \in \mathcal{T}$. However, $\lambda_{B}$ is not affine on generalized edges. Recall 


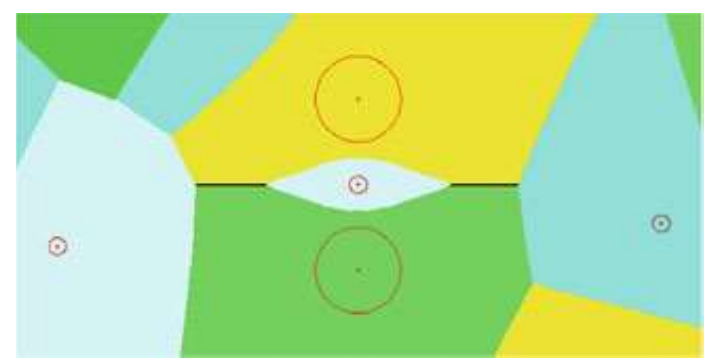

(a) Part of a Voronoi tessellation with a multiply connected Voronoi edge (black)

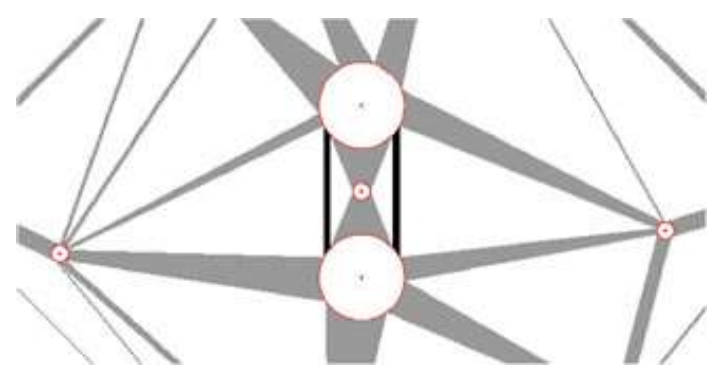

(b) Part of the generalized Delaunay triangulation with a multiply connected generalized edge (black shaded)

Fig. 4 Voronoi tessellation and Generalized Delaunay triangulation of a set of disks in the plane emphasizing possible non-connectivity of its elements

that a generalized edge $E \in \mathcal{E}$ is the agglomeration of line segments. The restriction of $\lambda_{B}$ to all those line segments is supposed to be affine. On the global level of the generalized edge $E$, this implies that $\left.\lambda_{B}\right|_{E}$ is the image of an affine function on the rectangular reference element $E^{\text {ref }}$ (cf. (2.3)) under the coordinate transformation $J_{E}$ (cf. (2.5)). After suitable rotation of the edge as in Sect. 2.3 (with $B_{1}=B$ ), $\left.\lambda_{B}\right|_{E}$ may be written as

$$
\lambda_{B}(x)=\left(1-\left(J_{E}^{-1}(x)\right)_{2}\right) \text { for all } x \in E
$$

where $\left(J_{E}^{-1}(x)\right)_{2}$ refers to the second component of the vector $J_{E}^{-1}(x)$.

Those basis functions generalize nodal basis functions on classical triangular meshes. In the special case of equally sized inclusions, those basis function have been used in the analysis of a network method [4]. The support of $\lambda_{B}$, denoted by $\omega_{B}$, is given by

$$
\omega_{B}:=B \cup(\cup\{E \in \mathcal{E}: E \cap B \neq \varnothing\}) \cup(\cup\{T \in \mathcal{T}: T \cap B \neq \emptyset\}) .
$$

Figure $3 \mathrm{~b}$ depicts a nodal basis function. Note that the set of nodal basis functions $\Lambda:=\left\{\lambda_{B}: B \in \mathcal{B}\right\}$ forms a partition of unity in $\Omega$. The generalized nodal basis functions which are not related to vertices on the Dirichlet boundary $\Gamma_{D}$ span the finite element space

$$
S^{\infty}:=\operatorname{span}(\Lambda) \cap V
$$

Obviously $S^{\infty}$ has dimension $\# \mathcal{B}$ which is minimal in comparison to data complexity and will be the space of choice for very large contrast and the special case of perfectly conducting inclusions $c_{\text {cont }}=\infty$. In the latter case the solution is necessarily constant with respect to every single inclusion (see Sect. 3.1), which is captured by $S^{\infty}$.

If $c_{\text {cont }}<\infty$ then the solution is not constant on the inclusions. Further basis functions (defined below) shall preserve sufficiently high accuracy in this setting, too. 
Fig. 5 Basis function $\lambda_{B}^{1}$

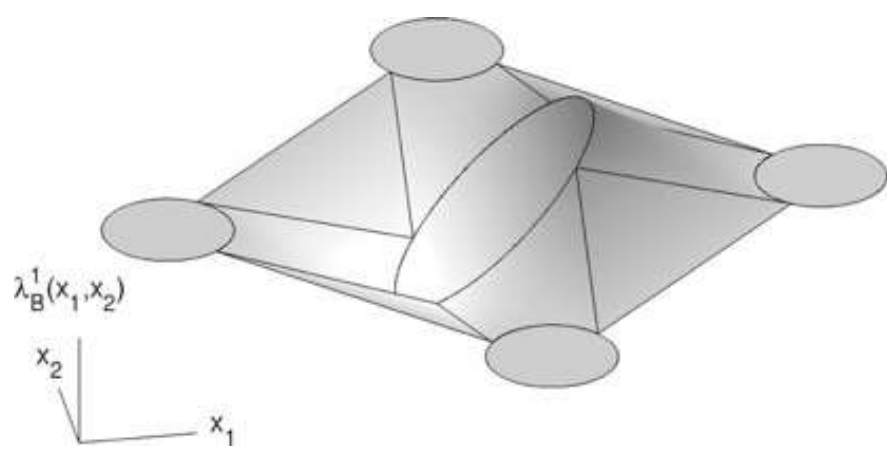

Every $B \in \mathcal{B}_{\text {inc }}$ defines (local) $\mathcal{T}_{\text {mat }}$-affine basis functions $\lambda_{B}^{1}, \lambda_{B}^{2}: \mathbb{R}^{2} \rightarrow[0,1]$ with

$$
\lambda_{B}^{k}(x)=\frac{x_{k}-\left(c_{B}\right)_{k}}{r_{B}} \quad \text { if } x \in B \text { while } \lambda_{B}^{k} \equiv 0 \text { in } \Omega_{\text {inc }} \backslash B
$$

The subscript $k$ refers to the $k$ th component of a 2-dimensional vector.

This means that $\lambda_{B}^{k}$ is affine on all inclusions $B \in \mathcal{B}_{\text {inc }}$ and all triangles $T \in \mathcal{T}$. After suitable rotation of the edge as in Sect. 2.3 (with $B_{1}=B$ ), $\left.\lambda_{B}\right|_{E}$ may be written as

$$
\lambda_{B}^{k}(x)=\left(1-\left(J_{E}^{-1}(x)\right)_{2}\right) \lambda_{B}^{k}\left(J_{E}\left(\left(J_{E}^{-1}(x)\right)_{1}, 0\right)\right) \text { for all } x \in E,
$$

where the coordinate transformation $J_{E}$ is given in (2.5). Note that $\left.J_{E}\left(\left(J_{E}^{-1}(\cdot)\right)_{1}, 0\right)\right) \in$ $\partial B$ and, hence, the values $\lambda_{B}^{k}\left(J_{E}\left(\left(J_{E}^{-1}(\cdot)\right)_{1}, 0\right)\right)$ are given by (2.4). It holds $\operatorname{supp}\left(\lambda_{B}^{k}\right)=\omega_{B}$. Figure 5 illustrates $\lambda_{B}^{1}$.

The enlarged finite element space is then given by

$$
S:=\operatorname{span}\left(\Lambda \cup\left\{\lambda_{B}^{1}: B \in \mathcal{B}\right\} \cup\left\{\lambda_{B}^{2}: B \in \mathcal{B}_{\text {inc }}\right\}\right) \cap V
$$

Remark 2 a) If the radii of all inclusions are zero, the spaces $S$ resp. $S^{\infty}$ reduce to the classical conforming $\mathbb{P}^{1}$ finite element space with respect to the Delaunay triangulation.

b) The number of degrees of freedom in $S$ is 3 per inclusion $B \in \mathcal{B}_{\text {inc }}$, and 1 per any other vertex $B \in \mathcal{B}_{\text {mat }}$ away from $\Gamma_{D}$. The overall number of degrees of freedom is bounded by $3 \# \mathcal{B}_{\text {inc }}+\# \mathcal{B}_{\text {mat }}$ and, hence, proportional to data complexity.

c) Further basis functions could easily be designed by considering any continuous function on $B$ and its $\mathcal{T}_{\text {mat }}$-affine or a more general $\mathcal{T}_{\text {mat }}$-polynomial extension to $\omega_{B}$.

\section{Galerkin approximation and a priori error analysis}

This section considers the variational formulation of (1.2) and its Galerkin approximation and presents error estimates which are independent of the contrast parameter $c_{\text {cont }}$. 
3.1 Variational formulation and solvability

Any minimizer $u^{*} \in \mathcal{A}$ of (1.2) solves the variational problem

$$
\int_{\Omega} c\left\langle\nabla u^{*}, \nabla v\right\rangle \mathrm{d} x=\int_{\Omega} f v \mathrm{~d} x \text { for all } v \in V
$$

The left-hand side of (3.1) defines a symmetric bilinear form $\mathfrak{a}$,

$$
\mathfrak{a}(u, v):=\int_{\Omega} c\langle\nabla u, \nabla v\rangle \mathrm{d} x
$$

The sum $u^{*}:=u+u_{D}$ is the solution of problem (3.1); $u_{D}$ denotes some extension (with finite energy) of the given inhomogeneous Dirichlet boundary data to $\Omega$. After shifting the inhomogeneous boundary data to the right-hand side, the problem reduces to find $u \in V$ such that

$$
\mathfrak{a}(u, v)=\int_{\Omega} f v \mathrm{~d} x-\mathfrak{a}\left(u_{D}, v\right)=: F(v) \text { for all } v \in V
$$

It is obvious that

$$
\frac{1}{1+C_{\mathrm{F}}}\|v\|_{H^{1}(\Omega)}^{2} \leq \mathfrak{a}(v, v) \quad \text { and } \quad \mathfrak{a}(u, v) \leq c_{\text {cont }}\|u\|_{H^{1}(\Omega)}\|v\|_{H^{1}(\Omega)}
$$

for all $u, v \in V$ with the constant from Friedrichs' inequality $C_{\mathrm{F}}$. Inequality (3.3) ensures the unique solvability of the variational problem (3.2) for finite contrast $c_{\text {cont }}<\infty$.

The Galerkin approximation of the solution of (3.2) with respect to the finite element space $S$, denoted by $u_{S} \in S$, is defined as the solution to the discrete variational system

$$
\mathfrak{a}\left(u_{S}, v\right)=F(v) \text { for all } v \in S
$$

Remark 3 a) The assembling of the corresponding linear system is fairly standard. It might be performed in a loop over all elements of the generalized finite element mesh (including triangles, disks, and edges), the computation of the local stiffness matrices and load vectors, and the sum of the local contributions to the global matrices. The computation of the entries of the local stiffness matrices might be done by transformation to the corresponding reference element. The only difficulty is that the transformation on the generalized edges is not affine. Still the entries of the local stiffness matrices might be precomputed as functions of the angle parameters and $\delta$. Alternatively, numerical quadrature can be used. If two inclusions are close to each other, the basis functions are close to be singular and the quadrature rule should take the singular behavior into account. 
b) The resulting stiffness matrix has a similar sparsity pattern as the stiffness matrix of the classical $P_{1}$ finite element method for the Poisson problem with respect to some regular triangulation. Hence, in the present 2-dimensional setting, sparse direct solvers offer robust, fast, and parallel solution of the linear system, even though the asymptotic complexity is not optimal (e.g. $\mathcal{O}\left(N^{3 / 2}\right)$ for nested dissection [15]). We refer to the textbook [9] for an overview on fast direct solvers for sparse linear systems. For moderate contrast, [1] and [2] show that an iterative solver based on hierarchical factorization performs almost optimal (i.e. $\left.\mathcal{O}\left(N(\log N)^{k}\right)\right)$. In the numerical examples in $[1,2]$, theses methods give promising results also in the high contrast regime.

This paper aims at a priori estimates of the error $u-u_{S}$ in energy norm $\|\cdot\|_{\mathfrak{a}}:=\sqrt{\mathfrak{a}(\cdot, \cdot)}$ and therefore estimates of the error in the effective conductivity. Since $u_{S}$ is the best approximation of $u$ in energy norm we have

$$
\begin{aligned}
2\left(\mathfrak{E}\left(u_{S}+u_{D}\right)-\mathfrak{E}\left(u+u_{D}\right)\right) & =\left\|\left(u+u_{D}\right)-\left(u_{S}+u_{D}\right)\right\|_{\mathfrak{a}}^{2} \\
& =\left\|u-u_{S}\right\|_{\mathfrak{a}}^{2}=\inf _{v \in S}\|u-v\|_{\mathfrak{a}}^{2}
\end{aligned}
$$

Sections 3.3 and 3.4 will present bounds of the right hand side in (3.5). A posteriori bounds are presented in [12].

\subsection{Perfectly conducting inclusions}

Our analysis shall cover the case of perfectly conducting inclusions as well. The related model is a variational problem with respect to the reduced space

$$
V^{\infty}:=\left\{v \in V:\left.v\right|_{B}=\text { const for all } B \in \mathcal{B}_{\text {inc }}\right\} \subset V
$$

We seek $u^{\infty} \in V^{\infty}$ such that

$$
\mathfrak{a}^{\infty}\left(u^{\infty}, v\right)=\int_{\Omega} f v \mathrm{~d} x-\mathfrak{a}^{\infty}\left(u_{D}^{\infty}, v\right)=: F(v) \text { for all } v \in V^{\infty}
$$

where $\mathfrak{a}^{\infty}(u, v):=\int_{\Omega_{\mathrm{mat}}}\langle\nabla u, \nabla v\rangle \mathrm{d} x$ for $u, v \in H^{1}(\Omega)$ and $u_{D}^{\infty} \in H^{1}(\Omega)$ with $\left.u_{D}^{\infty}\right|_{\Gamma_{D}}=\left.u_{D}\right|_{\Gamma_{D}}$ and $\left.\nabla u_{D}^{\infty}\right|_{B}=0$ for all $B \in \mathcal{B}_{\text {inc }}$.

Since $\mathfrak{a}^{\infty}(u, v) \leq\|u\|_{H^{1}(\Omega)}\|v\|_{H^{1}(\Omega)}$ and $\mathfrak{a}^{\infty}(v, v)=\|\nabla v\|_{L^{2}(\Omega)}^{2}$ for all $u, v \in$ $V^{\infty}$, the variational problem (3.6) has a unique solution.

The Galerkin approximation $u_{S} \in S^{\infty}$ of the solution of (3.6), with respect to the finite element space $S^{\infty}$ defined in (2.6), satisfies

$$
\mathfrak{a}^{\infty}\left(u_{S^{\infty}}, v\right)=F(v) \text { for all } v \in S^{\infty}
$$

The error estimate (3.5) remains valid with $u$ replaced by $u^{\infty}$ and $u_{S}$ replaced by $u_{S^{\infty}}$. 
Mathematical justification of the limiting problem We shall justify the model problem (3.6). For fixed geometry $\Omega_{\text {mat }}$, Dirichlet data $u_{D}=0$, and force term $f$, let $u_{c_{\text {cont }}}$ denote the solution of (3.2) associated with the contrast parameter $c_{\text {cont }} \geq 1$.

Define some function $\tilde{u}_{c_{\text {cont }}} \in V$ as follows. On every $B \in \mathcal{B}_{\text {inc }},\left.\left(\tilde{u}_{c_{\text {cont }}}\right)\right|_{B}$ equals $\left.u_{c_{\text {cont }}}\right|_{B}$ minus its mean value $|B|^{-1} \int_{B} u_{c_{\text {cont }}} \mathrm{d} x$. This defines $\left.\left(\tilde{u}_{c_{\text {cont }}}\right)\right|_{\Omega_{\text {inc }}}$. Observe that Poicareé's inequality yields $\left\|\tilde{u}_{c_{\text {cont }}}\right\|_{L^{2}\left(\Omega_{\text {inc }}\right)} \leq C_{1}\left\|\nabla \tilde{u}_{c_{\text {cont }}}\right\|_{L^{2}\left(\Omega_{\text {inc }}\right)}=$ $C_{1}\left\|\nabla u_{c_{\text {cont }}}\right\|_{L^{2}\left(\Omega_{\text {inc }}\right)}$ with some constant $C_{1}$ independent of contrast and the positions of the inclusions. In $\Omega_{\text {mat }}, \tilde{u}_{c_{\text {cont }}}$ is chosen as some bounded extension of $\left.\left(\tilde{u}_{c_{\text {cont }}}\right)\right|_{\Omega_{\text {inc }}}$ in the sense of [26], i.e., there is some constant $C_{2}$ that may depend on the geometry but not on $c_{\text {cont }}$ such that $\left\|\tilde{u}_{c_{\text {cont }}}\right\|_{H^{1}(\Omega)} \leq C_{2}\left\|u_{c_{\text {cont }}}\right\|_{H^{1}\left(\Omega_{\text {inc }}\right)}$.

This construction and the classical jump relation at the interface $\partial \Omega_{\text {inc }}$,

$$
c_{\text {cont }} \frac{\partial\left(\left.\left(u_{c_{\text {cont }}}\right)\right|_{\Omega_{\text {inc }}}\right)}{\partial \nu_{\Omega_{\text {inc }}}}=-\frac{\partial\left(\left.\left(u_{c_{\text {cont }}}\right)\right|_{\Omega_{\text {mat }}}\right)}{\partial \nu_{\Omega_{\text {mat }}}} \text { in } H^{-1 / 2}\left(\partial \Omega_{\text {inc }}\right) \text {, }
$$

yield

$$
\begin{aligned}
& \left\|\nabla u_{c_{\text {cont }}}\right\|_{L^{2}\left(\Omega_{\text {inc }}\right)} \\
& =\int_{\Omega_{\text {inc }}}\left\langle\nabla u_{c_{\text {cont }}}, \nabla \tilde{u}_{c_{\text {cont }}}\right\rangle \mathrm{d} x=\int_{\partial \Omega_{\text {inc }}} \frac{\partial u_{c_{\text {cont }}}}{\partial \nu_{\Omega_{\text {inc }}}} \tilde{u}_{c_{\text {cont }}} \mathrm{d} x+c_{\text {cont }}^{-1} \int_{\Omega_{\text {inc }}} f \tilde{u}_{c_{\text {cont }}} \mathrm{d} x \\
& \leq c_{\text {cont }}^{-1}\left(\left|\int_{\partial \Omega_{\mathrm{mat}}} \frac{\partial u_{c_{\mathrm{cont}}}}{\partial \nu_{\Omega_{\mathrm{mat}}}} \tilde{u}_{c_{\mathrm{cont}}} \mathrm{d} x\right|+\|f\|_{L^{2}\left(\Omega_{\mathrm{inc}}\right)}\left\|\tilde{u}_{c_{\mathrm{cont}}}\right\|_{L^{2}\left(\Omega_{\mathrm{inc}}\right)}\right) \\
& \leq c_{\text {cont }}^{-1}\left(\left\|\nabla u_{c_{\text {cont }}}\right\|_{L^{2}\left(\Omega_{\mathrm{mat}}\right)}\left\|\nabla \tilde{u}_{c_{\text {cont }}}\right\|_{L^{2}\left(\Omega_{\mathrm{mat}}\right)}+\|f\|_{L^{2}\left(\Omega_{\mathrm{mat}}\right)}\left\|\tilde{u}_{c_{\mathrm{cont}}}\right\|_{L^{2}\left(\Omega_{\mathrm{mat}}\right)}\right. \\
& \left.+\|f\|_{L^{2}\left(\Omega_{\text {inc }}\right)}\left\|\tilde{u}_{c_{\text {cont }}}\right\|_{L^{2}\left(\Omega_{\text {inc }}\right)}\right) \\
& \leq C c_{\text {cont }}^{-1}\|f\|_{L^{2}(\Omega)}\left\|\nabla u_{c_{\text {cont }}}\right\|_{L^{2}\left(\Omega_{\text {inc }}\right)} \text {, }
\end{aligned}
$$

where $C$ depends only on $C_{1}$ and $C_{2}$ but not on $c_{\text {cont }}$. This implies

$$
\left\|c^{1 / 2} \nabla u_{c_{\text {cont }}}\right\|_{L^{2}\left(\Omega_{\mathrm{inc}}\right)} \leq C c_{\mathrm{cont}}^{-1 / 2}\|f\|_{L^{2}(\Omega)}
$$

Hence, the solution $u_{c_{\text {cont }}}$ of (3.2) converges (with respect to the energy norm) to the solution $u^{\infty}$ of (3.6) as $c_{\text {cont }} \rightarrow \infty$.

\subsection{Nodal interpolation and approximability}

An upper bound for the right-hand side in (3.5) is derived through the design of some finite element function based on a suitable interpolation of the solution $u$. The conditions 


$$
\begin{aligned}
& \int_{B}(u-I u) v \mathrm{~d} x=0 \text { for all } v \in \mathbb{P}^{1}\left(\mathbb{R}^{2}\right) \text { and for all } B \in \mathcal{B}_{\text {inc }}, \\
& u(b)-I u(b)=0 \text { for all } B=\{b\} \in \mathcal{B}_{\text {mat }},
\end{aligned}
$$

define a generalized nodal interpolation operator $I: H^{2}\left(\Omega_{\text {mat }} \cup \Omega_{\text {inc }}\right) \rightarrow S_{0}$. Since, on any inclusion $B \in \mathcal{B}_{\text {inc }}, I u$ is the $L^{2}(B)$ projection of $u$ onto the space of affine functions, we have that

$$
\left\|\nabla^{m}(u-I u)\right\|_{L^{2}(B)} \leq C_{I} \operatorname{diam}(B)^{2-m}|u|_{H^{2}(B)} \text { for } m=0,1
$$

with some universal constant $C_{I}$ independent of the diameter of the disk $B$ and $u \in$ $H^{2}\left(\Omega_{\text {mat }} \cup \Omega_{\text {inc }}\right)$. The estimate (3.10) already provides approximation properties of the finite element space on the inclusions. It remains to give local estimates for the interpolation error on the triangles (see Lemma 3.1) and the generalized edges (see Lemma 3.3).

As usual, the error on a triangle $T$ depends on the aspect ratio $\rho_{T}$, i.e., the ratio between the diameters of the largest circle that can be inscribed in $T$ and the circumcircle of $T$.

Lemma 3.1 Let $u \in V \cap H^{2}\left(\Omega_{\text {mat }} \cup \Omega_{\text {inc }}\right)$ and let $T \in \mathcal{T}$ with vertices on $B_{1}, B_{2}, B_{3} \in$ $\mathcal{B}$. Then it holds

$$
\|\nabla(u-I u)\|_{L^{2}(T)}^{2} \leq C_{\mathcal{T}}^{2} \rho_{T}^{-2}\left\|h \nabla^{2} u\right\|_{L^{2}\left(T \cup B_{1} \cup B_{2} \cup B_{3}\right)}^{2}
$$

with some universal constant $C_{\mathcal{T}}$ which depends only on $C_{I}$ from (3.9).

Proof A key ingredient of the proof are standard estimates for the interpolation error with respect to a triangle $T$. It is well known (see [8, Theorem 16.1]) that the nodal (affine) interpolant $I_{T} u$ of $u$ at the vertices of $T$ satisfies

$$
\left|u-I_{T} u\right|_{H^{m}(T)} \leq C_{\mathrm{ip}} \rho_{T}^{-1} \operatorname{diam}(T)^{2-m}|u|_{H^{2}(T)} \quad \text { for all } u \in H^{2}(T), \quad m=0,1 .
$$

The difficulty is that $I u$ defined by (3.9) does not interpolate $u$ at the vertices of $T$ in general. Thus, the error is split into two components,

$$
\|\nabla(u-I u)\|_{L^{2}(T)}^{2} \leq\left\|\nabla\left(u-I_{T} u\right)\right\|_{L^{2}(T)}^{2}+\left\|\nabla\left(I_{T} u-I u\right)\right\|_{L^{2}(T)}^{2} .
$$

The first term on the right-hand side of (3.13) can be estimated directly with (3.12) while the second one requires further considerations.

Notice that $\left.\nabla\left(I_{T} u-I u\right)\right|_{T}$ is constant on $T$ and the inverse estimate

$$
\|\nabla q\|_{L^{\infty}(T)} \leq 2 \rho_{T}^{-1} \operatorname{diam}(T)^{-1}\|q\|_{L^{\infty}(T)}
$$


holds for all $q \in \mathbb{P}_{1}(T)$ on any triangle $T$. Thus

$$
\left\|\nabla\left(I_{T} u-I u\right)\right\|_{L^{2}(T)}^{2} \leq\left|T\left\|\left.\nabla\left(I_{T} u-I u\right)\right|_{L^{\infty}(T)} ^{2} \stackrel{(3.14)}{\leq} 4 \rho_{T}^{-2}\right\| I_{T} u-I u \|_{L^{\infty}(T)}^{2}\right.
$$

The maximal absolute value of the affine function $q:=\left.\left(I_{T} u-I u\right)\right|_{T}$ on $T$ is attained in some vertex $x_{0}=V(T) \cap B_{T}$ for some $B_{T} \in\left\{B_{1}, B_{2}, B_{3}\right\}$. If $B_{T} \in \mathcal{B}_{\text {mat }}$, i.e., $B_{T}=x_{0}$, then $\left.\left(I_{T} u-I u\right)\right|_{T}=0$. Otherwise, let $\tilde{T} \subset B_{T}$ be the equilateral triangle with vertices on $\partial B_{T}$ and one vertex at $x_{0}$. For $q \in \mathbb{P}_{1}(T)$ and $p \in \mathbb{P}_{1}(\tilde{T})$ with $\left|p\left(x_{0}\right)\right| \geq\left|q\left(x_{0}\right)\right|$ it holds

$$
\|q\|_{L^{\infty}(T)}^{2}=\left|q\left(x_{0}\right)\right|^{2} \leq\left|p\left(x_{0}\right)\right|^{2} \leq 2\left(|\tilde{T}|^{-1}\|p\|_{L^{2}(\tilde{T})}^{2}+\|\nabla p\|_{L^{2}(\tilde{T})}^{2}\right) .
$$

With the special choices $p=\left.\left(I_{T} u-I u\right)\right|_{T}$ and $q=\left.\left(I_{\tilde{T}} u-I u\right)\right|_{\tilde{T}}$ this leads to

$$
\begin{gathered}
\left\|\nabla\left(I_{T} u-I u\right)\right\|_{L^{2}(T)}^{2} \stackrel{(3.15),(3.16)}{\leq} 8 \rho_{T}^{-2}\left(|\tilde{T}|^{-1}\left\|I_{\tilde{T}} u-I u\right\|_{L^{2}(\tilde{T})}^{2}+\left\|\nabla\left(I_{\tilde{T}} u-I u\right)\right\|_{L^{2}(\tilde{T})}^{2}\right) \\
\stackrel{(3.12),(3.10)}{\leq} 16 \rho_{T}^{-2}\left(C_{I}^{2}+C_{\mathrm{ip}}^{2}\right) h_{B_{T}}^{2}\left\|\nabla^{2} u\right\|_{L^{2}\left(B_{T}\right)}^{2} .
\end{gathered}
$$

Together with (3.13) and (3.12) this implies (3.11) with $C_{\mathcal{T}}^{2} \leq 5\left(C_{I}+C_{\text {ip }}\right)$.

The second step of the error analysis considers the a priori estimate of the interpolation error on the generalized edges. Every connectivity component $E_{k}, k=$ $1,2, \ldots, K(E)$ of an edge $E \in \mathcal{E}$ is a curvilinear polygon, i.e., $E_{k}$ is a simplyconnected, bounded domain with the boundary $\partial E_{k}=\bigcup_{j=1}^{4} \tau_{j}$, where $\tau_{j}$ are circular arcs. Note that all internal angles $\gamma_{1}\left(E_{k}\right), \gamma_{2}\left(E_{k}\right), \ldots, \gamma_{4}\left(E_{k}\right)$ of $E_{k}$ are bounded from above by $\pi / 2$. The subsequent error analysis depends on the smallest angle which is denoted $\gamma_{E_{k}}$. Correspondingly, $\gamma_{E}:=\min _{k=1,2, \ldots, K(E)} \gamma_{E_{k}}$. The following lemma shows that all these angles are bounded from below by a positive constant.

Lemma 3.2 There exist $\gamma_{\mathcal{E}}>0$ such that $0<\gamma_{\mathcal{E}} \leq \gamma_{E}$ for all $E \in \mathcal{E}$.

Proof Let $E \in \mathcal{E}$ be some generalized edge connected to the inclusion $B \in \mathcal{B}_{\text {inc }}$. Let $\tau$ be one of the straight arcs that define the edge. By design, $\tau$ is an element of the infinite Delaunay triangulation $\mathcal{T}_{\text {mat }}$ (see Sect. 2.1). Since its circumdisk $C D(\tau)$ is tangential to $B$ (due to the Delaunay criterion (2.1)), $\tau$ by itself cannot be tangential to $B$ and the angle between $\tau$ and the circular arc $E \cap B$ is necessarily larger than zero.

Lemma 3.3 Let $u \in V \cap H^{2}\left(\Omega_{\text {mat }} \cup \Omega_{\text {inc }}\right)$ and let $E=E\left(B_{1}, B_{2}\right) \in \mathcal{E}$ be a generalized edge that connects two generalized vertices (inclusions) $B_{1}, B_{2} \in \mathcal{B}_{\text {inc. Then }}$

$$
\|\nabla(u-I u)\|_{L^{2}(E)}^{2} \leq C_{\mathcal{E}}\left(\left\|h \nabla^{2} u\right\|_{L^{2}(E)}^{2}+C_{E}\left\|h \nabla^{2} u\right\|_{L^{2}\left(B_{1} \cup B_{2}\right)}^{2}\right)
$$

holds with $C_{E}:=\max _{k=1,2}\left\|h_{B_{k}} / h+h / h_{B_{k}}\right\|_{L^{\infty}(E)}$ and some universal constant $C_{\mathcal{E}}$ which depends only on $\gamma_{E}$. 

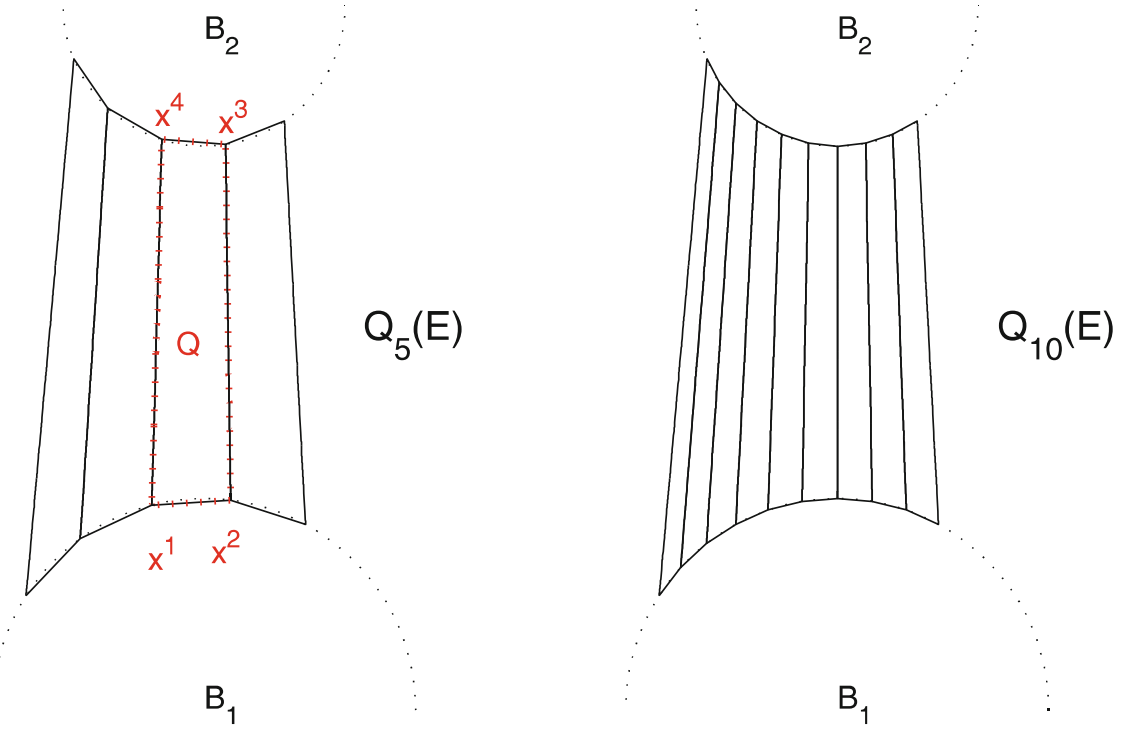

Fig. 6 Subdivisions $\mathcal{Q}_{5}(E)$ and $\mathcal{Q}_{10}(E)$ of some generalized edge $E=E\left(B_{1}, B_{2}\right) \in \mathcal{E}$ into quadrilaterals in the proof of Lemma 3.3

Proof The proof consists of two parts. Part $I$ proves the assertion for $c_{\text {cont }}=\infty$ and prepares the proof in the case $c_{\text {cont }}<\infty$ which is complemented in part $I I$.

Part I. Without loss of generality, let $E$ be connected, $r_{B_{1}} \geq r_{B_{2}}$, and $c_{B_{1}}=0$, $c_{B_{2}}=(0, \delta)$ for some $\delta>r_{B_{1}}+r_{B_{2}}$. The restriction $E \cap \partial B_{1}=\phi([\alpha, \beta])$ of $E$ to $B_{1}$ shall be parametrized by some angle $s \in[\alpha, \beta] \subset[-\pi / 2, \pi / 2]$ with $\phi(s):=$ $r_{B_{1}}(\sin (s), \cos (s))$. The parameter interval $[\alpha, \beta]$ is subdivided by equidistributed points

$$
\alpha=s^{1}<s^{2}<s^{3}<\cdots<s^{L}=\beta .
$$

These points are mapped by $\phi$ onto $B_{1}$ and by $\phi \circ \pi_{B_{1}}$ onto $B_{2}$ (recall (2.4) for the definition of $\pi_{B_{1}}$ ). Let

$$
\begin{aligned}
\mathcal{Q}_{L}(E) & :=\left\{Q_{\ell}: \ell=1, \ldots, L-1\right\} \text { with } \\
Q_{\ell} & :=\operatorname{conv}\left(\phi\left(s^{\ell}\right), \phi\left(s^{\ell+1}\right), \pi_{B_{1}}\left(\phi\left(s^{\ell+1}\right)\right), \pi_{B_{1}}\left(\phi\left(s^{\ell}\right)\right)\right)
\end{aligned}
$$

be a subdivision of $E$ into quadrilaterals (see Fig. 6).

The union of quadrilaterals on level $L$ provides a polygonal approximation $E^{L}:=$ $\bigcup_{Q \in \mathcal{Q}_{L}(E)} Q$ of $E \subset E^{L} \subset \operatorname{conv}(E)$ for all $L$ with $\left|E^{L} \backslash E\right| \rightarrow 0$ as $L \rightarrow \infty$. A (bounded) extension operator $(\cdot)_{E}: H^{2}(E) \rightarrow H^{2}\left(\mathbb{R}^{d}\right)$ (see, e.g., [26]) extends $\left.u\right|_{E}$ to $\operatorname{conv}(E)$. The extended function is denoted by $u_{E}$.

The nodal (bilinear) interpolation operator with respect to $Q \in \mathcal{Q}_{L}$ is denoted by $J_{Q}$ and its $\mathcal{Q}_{L}$-piecewise version by $J_{\mathcal{Q}_{L}}$. Theorem 3.8 from [22] implies

$$
\left\|\nabla\left(u_{E}-J_{Q} u_{E}\right)\right\|_{L^{2}(Q)} \leq C_{Q} \operatorname{diam}(Q)\left\|\nabla^{2} u_{E}\right\|_{L^{2}(Q)}
$$


for all $Q \in \mathcal{Q}_{L}, L \in \mathbb{N}$. The constant $C_{Q}$ depends only on the interior angles of $Q$, i.e., $C_{Q}$ can be bounded uniformly for all $Q \in \mathcal{Q}_{L}$ and all $L \in \mathbb{N}$ in terms of $\gamma_{E}$. Thus

$$
\begin{aligned}
&\left\|\nabla\left(u_{E}-J_{\mathcal{Q}_{L}} u_{E}\right)\right\|_{L^{2}\left(E^{L}\right)}^{2}=\sum_{Q \in \mathcal{Q}_{L}}\left\|\nabla\left(u_{E}-J_{Q} u_{E}\right)\right\|_{L^{2}(Q)}^{2} \\
& \stackrel{(3.18)}{\leq} \sum_{Q \in \mathcal{Q}_{L}} C_{1}\left\|\operatorname{diam}(Q) \nabla^{2} u_{E}\right\|_{L^{2}(Q)}^{2}
\end{aligned}
$$

with some constant $C_{1}$ which depends only on $\gamma_{E}$. Let $L$ tend to infinity in (3.19) to verify

$$
\|\nabla(u-\tilde{u})\|_{L^{2}(E)}^{2} \leq C_{1}\left\|h \nabla^{2} u\right\|_{L^{2}(E)}^{2}
$$

for $\tilde{u}:=\lim _{L \rightarrow \infty} J_{\mathcal{Q}_{L}} u_{E}$. If $c_{\text {cont }}=\infty$ then $\tilde{u}=I u$ and the proof is finished.

Part II. If otherwise $c_{\text {cont }}<\infty$ then, in general, $\tilde{u} \notin S$ and $\|\nabla(I u-\tilde{u})\|_{L^{2}(E)}$ needs to be estimated further. The sequence $e_{L}:=J_{\mathcal{Q}_{L}}(I u)_{E}-J_{\mathcal{Q}_{L}} u_{E}$ converges (in $\left.H^{1}\right)$ to $e:=I u-\tilde{u}$ as $L \rightarrow \infty$. Thus, bounds on $\left\|\nabla e_{L}\right\|_{L^{2}\left(E_{L}\right)}$ will lead to a bound on $\|\nabla(I u-\tilde{u})\|_{L^{2}(E)}$. Let $Q \in \mathcal{Q}_{L}$ with

$$
\partial Q=\left[x^{1}, x^{2}\right] \cup\left[x^{2}, x^{3}\right] \cup\left[x^{3}, x^{4}\right] \cup\left[x^{4}, x^{1}\right]
$$

and $x^{1}, x^{2} \in B_{1}$ and $x^{3}, x^{4} \in B_{2}$ and $x^{5}=x^{1}$ as in Fig. 6 (left). The vector $\left.\nabla e_{L}\right|_{Q}$ is written as some linear combination of the vectors $\left(x^{k+1}-x^{k}\right) /\left|x^{k+1}-x^{k}\right|$ such that

$$
\left\|\nabla e_{L}\right\|_{L^{2}(Q)}^{2} \leq|Q|\left\|\nabla e_{L}\right\|_{L^{\infty}(Q)}^{2} \leq C_{2}|Q| \sum_{k=1}^{4} \frac{\left|\left\langle\left.\left(\nabla e_{L}\right)\right|_{\left[x^{k}, x^{k+1}\right]}, x^{k+1}-x^{k}\right\rangle\right|^{2}}{\left|x^{k+1}-x^{k}\right|^{2}}
$$

with a constant $C_{2}$ which depends only on the maximal angle in $Q$ and can be bounded uniformly in terms of $\gamma_{E}^{-1}$. Using $\left\langle\left.\left(\nabla e_{L}\right)\right|_{\left[x^{k}, x^{k+1}\right]}, x^{k+1}-x^{k}\right\rangle=e_{L}\left(x^{k+1}\right)-e_{L}\left(x^{k}\right)$ for $k=2$ and $k=4$, this yields

$$
\begin{aligned}
\left\|\nabla e_{L}\right\|_{L^{2}(Q)}^{2} \leq & C_{2}\left(\|h\|_{L^{\infty}(Q)}\left\|\nabla e_{L}\right\|_{L^{2}\left(\left[x^{1}, x^{2}\right] \cup\left[x^{3}, x^{4}\right]\right)}^{2}\right. \\
& \left.+\left\|h^{-1}\right\|_{L^{\infty}(Q)}\|e\|_{L^{2}\left(\left[x^{2}, x^{3}\right] \cup\left[x^{4}, x^{1}\right]\right)}^{2}\right) .
\end{aligned}
$$

The summation of (3.21) over $Q \in \mathcal{Q}_{L}$ leads to

$$
\begin{aligned}
\left\|\nabla e_{L}\right\|_{L^{2}\left(E_{L}\right)}^{2} \leq & C_{2}\left(\|h\|_{L^{\infty}(E)}\left\|\nabla e_{L}\right\|_{L^{2}\left(\partial E_{L} \cap\left(B_{1} \cup B_{2}\right)\right)}^{2}\right. \\
& \left.+\left\|h^{-1}\right\|_{L^{\infty}(E)}\left\|e_{L}\right\|_{L^{2}\left(\partial E_{L} \cap\left(B_{1} \cup B_{2}\right)\right)}^{2}\right) .
\end{aligned}
$$


In the limit $L \rightarrow \infty$ it follows

$$
\|\nabla e\|_{L^{2}(E)}^{2} \leq C_{2}\left(\|h\|_{L^{\infty}(Q)}\|\nabla e\|_{L^{2}\left(\partial E \cap\left(B_{1} \cup B_{2}\right)\right)}^{2}+\left\|h^{-1}\right\|_{L^{\infty}(Q)}\|e\|_{L^{2}\left(\partial E \cap\left(B_{1} \cup B_{2}\right)\right)}^{2}\right) .
$$

Estimate (3.10) and the trace inequality

$$
\|f\|_{L^{2}(\partial B)} \leq \sqrt[4]{8}\left(\|f\|_{L^{2}(B)}+\|f\|_{L^{2}(B)}^{1 / 2}\|\nabla f\|_{L^{2}(B)}^{1 / 2}\right)
$$

valid for any disk $B$ and $f \in H^{1}(B)$ (see [6, Proposition 1.6.3]), imply

$$
\begin{aligned}
& |e|_{H^{m}(\partial B)}=\quad|\tilde{u}-I u|_{H^{m}(\partial B)}=|u-I u|_{H^{m}(\partial B)} \\
& \stackrel{(3.23),(3.10)}{\leq} \sqrt[4]{8} C_{I} r_{B}^{2-m-\frac{1}{2}}|u|_{H^{2}(B)} \text { for } m=0,1 .
\end{aligned}
$$

With a universal constant $C_{3}$ which depends only on $C_{I}$ and $\gamma_{E}$ (through $C_{1}$ and $C_{2}$ ), this leads to

$$
\|\nabla(I u-\tilde{u})\|_{L^{2}(E)}^{2} \stackrel{(3.21),(3.24)}{\leq} C_{3}\left\|h^{-1}\left(\left.h\right|_{B_{k}}\right)+h\left(\left.h\right|_{B_{k}}\right)^{-1}\right\|_{L^{\infty}(E)}\left\|h \nabla^{2} u\right\|_{H^{2}\left(B_{1} \cup B_{2}\right)}^{2} .
$$

This concludes the proof of the lemma.

The constant $C_{E}$ reflects the fact that two inclusions might touch but the corresponding affine approximations of the solution on the disks might not match at the touching point. Thus, in rare cases for $c_{\text {cont }}<\infty$, the discrete system might have infinite energy whereas the continuous solution has not. Choosing sufficiently many degrees of freedom (number of degrees of freedom per inclusion larger than or equal to the number of neighbors per inclusion) this problem disappears.

\subsection{A priori error estimates}

The approximation property of the finite element space $S$ reads as follows.

Theorem 3.1 Let $u \in V \cap H^{2}\left(\Omega_{\text {mat }} \cup \Omega_{\text {inc }}\right)$ be the solution of (3.2) and let $u_{S} \in S$ be its Galerkin approximation that solves (3.4). Then it holds

$$
\left\|u-u_{S}\right\|_{\mathfrak{a}}^{2} \leq C_{S}^{2}\left(\left\|h \nabla^{2} u\right\|_{L^{2}\left(\Omega_{\mathrm{mat}}\right)}^{2}+c_{\mathrm{cont}} \sum_{B \in \mathcal{B}_{\mathrm{inc}}} C_{B}\left\|h \nabla^{2} u\right\|_{L^{2}(B)}^{2}\right)
$$

with $C_{B}:=\left\|h_{B} / h+h / h_{B}\right\|_{L^{\infty}{ }_{\left(\omega_{B}\right)}}$ and some universal constant $C_{S}$ which depends only on $C_{I}, C_{\mathcal{T}}$, and $C_{\mathcal{E}}$. 
Proof The proof is a straight forward consequence of (3.10), Lemma 3.1, Lemma 3.3, and the equality

$$
\|v\|_{\mathfrak{a}}^{2}=\|\nabla v\|_{L^{2}\left(\Omega_{\mathrm{mat}}\right)}^{2}+c_{\mathrm{cont}}\|\nabla v\|_{L^{2}\left(\Omega_{\mathrm{inc}}\right)}^{2} \text { for all } v \in H^{1}(\Omega) .
$$

By (3.3) the estimate of Theorem 3.1 is also valid for the error measured in the $H^{1}(\Omega)$-norm. The regularity results from [7, Appendix B] read

$$
\left\|\nabla^{2} u\right\|_{L^{2}\left(\Omega_{\mathrm{mat}}\right)} \leq C_{\mathrm{reg}}\|f\|_{L^{2}(\Omega)}, \quad\left\|\nabla^{2} u\right\|_{L^{2}\left(\Omega_{\mathrm{inc}}\right)} \leq \frac{C_{\mathrm{reg}}}{c_{\mathrm{cont}}}\|f\|_{L^{2}(\Omega)} .
$$

The constant $C_{\text {reg }}$ depends solely on the geometry of the set inclusions and $\Omega$ but not on $c_{\text {cont }}$. This implies that the contrast is not a critical parameter.

Corollary 3.1 Let $u \in V \cap H^{2}\left(\Omega_{\text {mat }} \cup \Omega_{\text {inc }}\right)$ be the solution of (3.2) and $u_{S} \in S$ its Galerkin approximation that solves (3.4). Then it holds

$$
\left\|u-u_{S}\right\|_{\mathfrak{a}} \leq \tilde{C}_{S}\|h\|_{L^{\infty}(\Omega)}\left(\|f\|_{L^{2}(\Omega)}+\left\|\nabla u_{D}\right\|_{L^{2}(\Omega)}\right)
$$

with some universal constant $\tilde{C}_{S}$ which depends only on $C_{\mathrm{reg}}$ and the constants $C_{S}, C_{B}$ from Theorem 3.1.

The constant $\tilde{C}_{S}$ in (3.26) does not depend on the contrast parameter $c_{\text {cont }}>1$. However, through the constants $C_{B}$, it might depend on the term (cf. the Definition of $C_{B}$ in Theorem 3.1)

$$
\max _{E\left(B_{1}, B_{2}\right) \in \mathcal{E}} \frac{\max \left\{r_{B_{1}}, r_{B_{2}}\right\}}{\operatorname{dist}\left(B_{1}, B_{2}\right) c_{\text {cont }}} .
$$

The latter constant is critical with regard to the geometry of the coefficient function. The term may blow up, whenever the distance of two inclusions relative to their size becomes very small. However, high contrast reduces this effect. In the case of perfectly conducting inclusions $\left(c_{\text {cont }}=\infty\right)$ it even disappears. The generalized interpolation operator from (3.9) fulfills $\left.(u-I u)\right|_{B}=0$ for all $B \in \mathcal{B}$ and the proof of Lemma 3.3 consists only of part $I$. Lemma 3.1 can be simplified in a similar way which leads to the following corollary.

Corollary 3.2 Let $c_{\text {cont }}=\infty$ and let $u^{\infty} \in V^{\infty} \cap H^{2}\left(\Omega_{\text {mat }} \cup \Omega_{\text {inc }}\right)$ be the solution of (3.6) and $u_{S^{\infty}} \in S^{\infty}$ its Galerkin approximation that solves (3.7). Then it holds

$$
\left\|\nabla\left(u^{\infty}-u_{S^{\infty}}\right)\right\|_{L^{2}(\Omega)}=\left\|u^{\infty}-u_{S^{\infty}}\right\|_{\mathfrak{a}} \leq C_{S^{\infty}}\left\|h \nabla^{2} u^{\infty}\right\|_{L^{2}\left(\Omega_{\mathrm{mat}}\right)}
$$

with a constant $C_{S^{\infty}}$ independent of $u^{\infty}, c_{\mathrm{cont}}$, and the location of the inclusions. 
In the general case $c_{\text {cont }}<\infty$ the critical constant shown in (3.27) can easily be reduced with higher-order ansatz functions on the inclusions. We can therefore derive error estimates whose constants are explicit in the underlying geometry. However, in all cases the dependence on the $H^{2}$-norm of the solution remains. This issue is briefly discussed in the Sect. 4.3.

\section{Concluding remarks}

The main result of this paper is a numerical scheme to compute temperature distributions in composite materials with a large number of particles and high contrast. In the model situation under consideration, the method is robust and does not depend on the contrast $c_{\text {cont }} \rightarrow \infty$. Some of the results extend to a more general geometric setting in a straight-forward way. However, some difficulties remain open.

\subsection{General inclusion geometry}

For the use in practical applications it is desirable to incorporate more general inclusion shapes and 3-dimensional geometries. It is shown in [23] that the generalized partitions of Sect. 2 nicely generalize to sets of convex inclusions, e.g., ellipsoids, convex polyhedra, and line segments. Even more, the design allows inclusions to intersect. Thus, generalized Delaunay triangulations are also available for non-convex inclusions which can be represented by finite unions of convex ones. The design of according finite element methods can be done similarly as presented here. However, the complexity of the mesh and the corresponding finite element method will grow as the number of shape parameters that define a single inclusion grows. For smooth inclusions the corresponding analysis is straight-forward; non-smooth inclusions, however, require new arguments which are able to cope with lack of regularity.

\subsection{Convergence}

By straight forward arguments it is easy to show that the finite element solutions (the solutions of (3.2) and (3.6)) converge in $H^{1}$ to the solution of (1.2) if the meshwidth function $h$ tends to 0 .

In the matrix, the meshwidth function $h$ can be decreased in the matrix $\Omega_{\text {mat }}$ by simply putting additional artificial inclusions (points) in the set $\mathcal{B}_{\text {mat }}$. If $c_{\text {cont }}=\infty$, this suffices to be able to construct a convergent sequence of approximation because the (energy-)error in the inclusions is always zero. The case, in which additional vertices of radius zero are added to improve the approximability properties of the finite element space, is already treated by the theory presented in this article. A different possibility is to leave the initial partition as it is and increase the polynomial degree of the shape functions. This strategy, the so-called $p$-refinement, is recommended for problems where geometry and data are smooth. The definition of higher-order finite element spaces is to some extent straight-forward, the corresponding analysis, however, appears more involved.

If $c_{\text {cont }}<\infty$, in addition, the error on the inclusions has to be decreased, e.g., by increasing the polynomial degree. 


\subsection{Geometry-explicit estimates}

The method presented is stable with respect to contrast in the medium. However, the error bounds might depend on geometric parameters of the material, e.g., the distance between neighboring particles. Whether or not the dependence on the local distance is critical depends on the global distribution of particles. This can be seen already in the simplified situation of perfectly conducting $\left(c_{\text {cont }}=\infty\right)$ inclusions.

Consider first two inclusions that touch but are isolated from further inclusions. Since the solution is found in $H^{1}$ the (constant) values of the solution on the two inclusions have to be equal. Provided the force term is sufficiently smooth $\left(L^{2}\right)$, classical regularity theory ensures smoothness of the solution in some neighborhood of the two inclusions and the constant in the regularity estimate depends only on the distance to further inclusions or the boundary of the domain.

The critical scenario is the appearance of an almost conducting path of inclusions which connects two parts of the outer boundary with different, prescribed temperature. The temperature gap needs to be compensated in the small regions between the inclusions of the path which might cause steep gradients in the solution. If the inclusions of the path touch pairwise, the path is perfectly conducting and hence, the energy is infinite. Depending on the volume fraction of particles, the material shows a phase transition from moderate to high conductivity. Mathematically speaking, the solution operator, which maps a pair the date $u_{D}$ and $f$ to the solution of (1.2), is not uniformly bounded with respect to the geometry of the set of inclusions $\mathcal{I}$ [25, Theorem 3.5] shows that, though the energy of the solution might blow up, the error estimate in Corollary 3.2 is bounded by some generic constant independent of the distance of the particles. Thus, our method is robust with respect the such critical scenarios and allows meaningful material simulation even in densely packed composites. We refer to [12] for numerical experiments.

In the general case of high but finite contrast the situation appears more involved and a corresponding regularity theory that is explicit (and sharp) with respect to both, contrast and geometric parameters, is not yet available and has to be addressed in future research.

\section{References}

1. Bebendorf, M.: Why finite element discretizations can be factored by triangular hierarchical matrices. SIAM J. Numer. Anal. 45(4), 1472-1494 (2007)

2. Börm, S.: Approximation of solution operators of elliptic partial differential equations by $\mathcal{H}$ - and $\mathcal{H}^{2}$-matrices. Numer. Math. 115(2), 165-193 (2010)

3. Berlyand, L., Kolpakov, A.: Network approximation in the limit of small interparticle distance of the effective properties of a high-contrast random dispersed composite. Arch. Ration. Mech. Anal. 159(3), 179-227 (2001)

4. Berlyand, L., Novikov, A.: Error of the network approximation for densely packed composites with irregular geometry. SIAM J. Math. Anal. 34(2), 385-408 (2002) (electronic)

5. Borcea, L., Papanicolaou, G.C.: Network approximation for transport properties of high contrast materials. SIAM J. Appl. Math 58, 501-539 (1998)

6. Brenner, S.C., Scott, L.R.: The mathematical theory of finite element methods. In: Texts in Applied Mathematics, 3rd edn, vol. 15. Springer, New York (2008) 
7. Chu, C.-C., Graham, I.G., Hou, T.Y.: A new multiscale finite element method for high-contrast elliptic interface problems. Math. Comput. 79, 1915-1955 (2010)

8. Ciarlet, P.: The Finite Element Method for Elliptic Problems. North Holland, Amsterdam (1978)

9. Davis, T.A.: Direct methods for sparse linear systems. In: Fundamentals of Algorithms, vol. 2. Society for Industrial and Applied Mathematics (SIAM), Philadelphia (2006)

10. Delaunay, B.: Sur la sphère vide. Izvestia Akademii Nauk SSSR, Otdelenie Matematicheskikh i Estestvennykh Nauk 7, 793-800 (1934)

11. Weinan, E., Engquist, B.: The heterogeneous multiscale methods. Commun. Math. Sci. 1(1), 87-132 (2003)

12. Eigel, M., Peterseim, D.: Network FEM for Composite Materials with A Posteriori Control DFG Research Center Matheon Berlin, Preprint Series, vol. 985 (2012)

13. Fortune, S.: A sweepline algorithm for Voronor diagrams. Algorithmica 2(2), 153-174 (1987)

14. Gavrilova, M., Rokne, J.: Swap conditions for dynamic Voronoi diagrams for circles and line segments. Comput. Aided Geom. Design 16(2), 89-106 (1999)

15. George, A., Liu, J.: Computer Solution of Large Sparse Positive Definite Systems. Prentice-Hall, Englewood Cliffs (1981)

16. Hou, T.Y., Wu, X.-H.: A multiscale finite element method for elliptic problems in composite materials and porous media. J. Comput. Phys. 134, 169-189 (1997)

17. Hughes, T.J.R., Feijóo, G.R., Mazzei, L., Quincy, J.-B.: The variational multiscale method-a paradigm for computational mechanics. Comput. Methods Appl. Mech. Eng. 166(1-2), 3-24 (1998)

18. Kim, D.-S., Kim, D., Sugihara, K.: Voronoi diagram of a circle set from Voronoi diagram of a point set. I. Topology. Comput. Aided Geom. Design 18(6), 541-562 (2001)

19. Kolpakov, A.A., Kolpakov, A.G.: Capacity and transport in contrast composite structures. CRC Press, Boca Raton (2010)

20. Larson, M.G., Målqvist, A.: Adaptive variational multiscale methods based on a posteriori error estimation: energy norm estimates for elliptic problems. Comput. Methods Appl. Mech. Eng. 196(21-24), 2313-2324 (2007)

21. Målqvist, A., Peterseim, D.: Localization of Elliptic Multiscale Problems. ArXiv e-prints, 1110.0692 (2011)

22. Mao, S., Nicaise, S., Shi, Z.-C.: On the interpolation error estimates for $Q_{1}$ quadrilateral finite elements. SIAM J. Numer. Anal. 47(1), 467-486 (2008)

23. Peterseim, D.: Generalized Delaunay partitions and composite material modeling. DFG Research Center Matheon Berlin, Preprint Series, vol. 690 (2010)

24. Peterseim, D.: Triangulating a system of disks. In: Proceedings of the EuroCG 2010. Dortmund, Germany (2010)

25. Peterseim, D.: Robustness of Finite Element Simulations in Densely Packed Random Particle Composites. Netw. Heterog Media 7(1), 113-126 (2012)

26. Stein, E.M.: Singular Integrals and Differentiablity Properties of Function. Priceton Univ. Press, New York (1970)

27. Voronoi, G.F.: Nouvelles applications des paramètres continus à la théorie des formes quadratiques. Journal für die Reine und Angewandte Mathematik 133, 97-178 (1907) 